\title{
Prevalência de sobrepeso/obesidade em crianças e adolescentes com constipação crônica funcional
}

\author{
Overweight/obesity prevalence in children and adolescents with chronic functional constipation
}

Francisca Teresa V. Faleiros ${ }^{1}$, Cristiane Camargo Omae², Cristiane Yoshie Nakazawa², Mary de Assis Carvalho ${ }^{3}$ Nilton Carlos Machado ${ }^{4}$

\section{RESUMO}

Objetivo: Avaliar a prevalência do sobrepeso/obesidade em um grupo de crianças com constipação crônica funcional.

Métodos: Estudo retrospectivo com 257 crianças de dois a 15 anos com constipação crônica funcional diagnosticada segundo critérios de Roma II, atendidas consecutivamente em clínica terciária de gastroenterologia pediátrica. Informações demográficas, clínicas, diagnósticas e antropométricas foram obtidas de prontuários. $\mathrm{O}$ índice de massa corporal (IMC) foi calculado e utilizou-se a referência do Centers for Disease Control para determinar o percentil de IMC para idade e gênero. Crianças com percentil de IMC $\geq 85$ foram consideradas com sobrepeso/ obesidade; as com percentis $\geq 5$ e $<85$ foram classificadas como eutróficas.

Resultados: Para as variáveis sociodemográficas e clínicas, não houve diferença estatisticamente significante entre os subgrupos sobrepeso/obesidade e eutrofia. A prevalência de crianças constipadas com sobrepeso/obesidade foi de 24,4\%. A média dos percentis do subgrupo sobrepeso/ obesidade foi $94,4 \pm 4,3$; a das eutróficas foi $45,3 \pm 24,2$. A prevalência de sobrepeso/obesidade foi similar em ambos os sexos e grupos etários ( $<7$ anos e $\geq 7$ anos).

Conclusões: Os subgrupos dos eutróficos e sobrepeso/obesidade foram homogêneos nas características sociodemográficas e clínicas, sugerindo que tais variáveis não influenciaram na prevalência do excesso de peso. A prevalência estimada

Instituição: Departamento de Pediatria da Faculdade de Medicina de Botucatu da Universidade Estadual Paulista (Unesp), Botucatu, SP, Brasil ${ }^{1}$ Professora-assistente, doutora da disciplina Pediatria Social do Departamento de Pediatria da Faculdade de Medicina de Botucatu da Unesp, Botucatu, SP, Brasil

${ }^{2}$ Graduanda da Faculdade de Medicina de Botucatu da Unesp, Botucatu, SP, Brasil

${ }^{3}$ Médica da disciplina Gastroenterologia Pediátrica do Departamento de Pediatria da Faculdade de Medicina de Botucatu da Unesp, Botucatu, SP, Brasil

${ }^{4}$ Professor-assistente, doutor da disciplina Gastroenterologia Pediátrica do Departamento de Pediatria da Faculdade de Medicina de Botucatu da Unesp, Botucatu, SP, Brasil de sobrepeso/obesidade entre as crianças com constipação crônica funcional está dentro dos valores esperados para a população pediátrica brasileira.

Palavras-chave: obesidade; sobrepeso; constipação intestinal; epidemiologia.

\section{ABSTRACT}

Objective: To determine the prevalence of overweight/ obesity in a group of children with chronic functional constipation.

Methods: Retrospective chart review of 257 children with chronic functional constipation as defined by Rome II criteria, 2-15 years old, assisted in a tertiary pediatric gastroenterology clinic. Demographic, clinical, diagnostic and anthropometric information was extracted from the charts. Body mass index (BMI) was calculated and The Centers for Disease Control's BMI growth reference was used to determine age and gender-specific BMI percentiles of the children. Overweight/obesity was classified as a BMI of $\geq 85^{\text {th }}$ percentile, and euthrophy as BMI $\geq 5^{\text {th }}$ and $<85^{\text {th }}$ percentile.

Results: There was no statistically significant difference for demographics and clinical variables between the subgroups overweight/obesity and eutrophy. In constipated children, prevalence of overweight/obesity was $24.4 \%$. Prevalence of overweight/obesity did not differ

Endereço para correspondência:

Nilton Carlos Machado

Departamento de Pediatra da Faculdade de Medicina de Botucatu

Campus Universitário Rubião Júnior

CEP 18618-970 - Botucatu/SP

E-mail: nmachado@fmb.unesp.br

Recebido em: 28/3/2008

Aprovado em: 26/7/2008 
between boys and girls or across age groups ( $<7$ years and $\geq 7$ years).

Conclusions: Demographics and clinical characteristics were similar between the overweight/obese and eutrophic subgroups, which suggests that these characteristics do not influence on the prevalence of overweight/obesity in constipated children. The estimated prevalence of overweight/ obesity in this group was similar to the prevalence of agematched reference for Brazilian children.

Key-words: obesity; overweight; constipation; epidemiology.

\section{Introdução}

$\mathrm{Na}$ clínica pediátrica, a constipação crônica funcional e o sobrepeso/obesidade são problemas freqüentes. A constipação crônica está entre os dez problemas mais comuns na prática pediátrica geral $^{(1)}$ e sua prevalência varia de 14,5 a $38,4 \%{ }^{(2)}$, o que representa cerca de $30 \%$ das consultas em um serviço terciário de Gastroenterologia Pediátrica ${ }^{(3)}$.

A prevalência de sobrepeso/obesidade em crianças tem apresentado um aumento ${ }^{(4-6)}$, sendo esse um grande desafio para o Pediatra, pois se associa a múltiplos problemas crônicos de saúde como diabetes, hiperlipidemias, hipertensão arterial, esteatose hepática e problemas psicossociais ${ }^{(7)}$.

Estudos mostram que há associação entre obesidade e sintomas gastrointestinais ${ }^{(8)}$ e, especialmente, constipação no caso dos adultos ${ }^{(9-10)}$. No que diz respeito às crianças, são poucas as publicações que avaliam tal associação. Em pesquisa na base de dados Medline a partir dos termos obesity, chronic constipation e childhood, encontrou-se, no período de 1990 a 2007, três estudos de prevalência da relação entre constipação funcional e obesidade. Fishman et al ${ }^{(11)}$ avaliaram a prevalência de constipação em 80 crianças obesas com idade entre um e 18 anos, das quais $23 \%$ eram constipadas, estando o soiling presente em $15 \%$ delas. Entretanto, neste estudo não houve um grupo controle. Pashankar e Loening-Baucke ${ }^{(12)}$ avaliaram a prevalência da obesidade em 719 crianças com idades entre quatro e 18 anos com constipação funcional, constatando prevalência significativamente elevada de obesidade neste grupo $(22,4 \%)$ em relação ao controle $(11,7 \%)$. Na comparação das crianças com e sem escape fecal, não foram observadas diferenças na prevalência da obesidade. Misra $e t$ $a l^{(13)}$ analisaram 101 crianças com constipação e encontraram maior prevalência de sobrepeso neste grupo (44\%), quando comparado ao controle (30\%).
Devido à alta prevalência destes dois problemas na prática pediátrica, à sua cronicidade, a dificuldades na abordagem e à necessidade de planejamento terapêutico e seguimento prolongado, considerou-se importante avaliar, em nosso meio, a relação entre ambos. Dessa forma, este estudo visa avaliar a prevalência do sobrepeso/obesidade em crianças com constipação crônica funcional em uma clínica de Gastroenterologia Pediátrica de nível terciário e comparar variáveis demográficas e clínicas entre crianças com sobrepeso/obesidade e aquelas com índice de massa corporal (IMC) normal.

\section{Métodos}

Estudo observacional retrospectivo realizado a partir de uma casuística de 257 crianças e adolescentes com constipação crônica funcional, atendidas consecutivamente e catalogadas em um Banco de Dados da disciplina de Gastroenterologia Pediátrica do Hospital das Clínicas da Faculdade de Medicina da Universidade Estadual Paulista de Botucatu, feito no período de janeiro de 2000 a dezembro de 2004. Foram utilizadas informações dos prontuários referentes à primeira consulta. Constipação crônica funcional foi definida, segundo os critérios de Roma II, como pelo menos duas semanas de fezes cibalosas, empedradas, endurecidas na maioria das evacuações ou fezes endurecidas, duas ou menos vezes por semana sem evidência de doença estrutural, endócrina ou metabólica ${ }^{(14)}$. Foi critério de inclusão a idade (entre dois e 15 anos) e de exclusão outros problemas crônicos de saúde, tais como, doenças neurológicas, genéticas, mentais ou distúrbios do crescimento e desenvolvimento e casos de constipação de etiologia orgânica.

Foram estudadas as variáveis demográficas (idade, gênero, idade e instrução dos pais, número de cômodos, pessoas e crianças no local de moradia) e clínicas (idade da primeira consulta, tempo de sintomas). O peso e a estatura foram avaliados por enfermeiras experientes no momento da primeira consulta. Para a aferição do peso (em kg), foi utilizada uma balança tipo plataforma, da marca Filizola ${ }^{\circledR}$ (carga máxima de $150 \mathrm{~kg}$ e precisão de $100 \mathrm{~g}$ ) e para a aferição da estatura $(\mathrm{em} \mathrm{cm})$, um antropômetro vertical segundo técnica descrita por Barros e Victora ${ }^{(15)}$. O IMC e o percentil do IMC foram calculados a partir do Programa Epi-Info $2002^{(16)}$. As crianças e adolescentes foram classificadas em três subgrupos, de acordo com o IMC: desnutridas (IMC $<5$ ), eutróficas $(\geq 5 \mathrm{IMC}<85)$ e sobrepeso/obesidade $(\mathrm{IMC} \geq 85)^{(17)}$. 
Os testes $t$ de Student e exato de Fisher foram utilizados para comparar as variáveis entre os subgrupos: pacientes eutróficos e com sobrepeso/obesidade. Um valor de $p<0,05$ foi considerado significativo. O programa estatístico utilizado para todos os cálculos foi o GraphPad Prism Version 3.0, $1999^{(18)}$. O estudo foi aprovado pelo Comitê de Ética em Pesquisa da Faculdade de Medicina da Universidade Estadual Paulista de Botucatu.

\section{Resultados}

Foram incluídas 257 crianças e adolescentes, sendo 151 $(58 \%)$ menores de sete anos e 106 (42\%) maiores. O grupo sobrepeso/obesidade era formado por 65 crianças, sendo 31 (48\%) com sobrepeso e $34(52 \%)$ com obesidade. Não foram observadas diferenças em relação às variáveis demográficas e clínicas entre as crianças e adolescentes com sobrepeso/ obesidade e as eutróficas (Tabela 1).

A distribuição de crianças e adolescentes segundo o percentil do IMC foi: $6,2 \%$ desnutridas; $69,4 \%$ eutróficas e $24,4 \%$ com sobrepeso/obesidade. Não foram observadas diferenças significantes quanto à prevalência de sobrepeso/obesidade entre menores e maiores de sete anos de idade (teste exato de Fisher, $p=0,54$ ). A média dos percentis de IMC do subgrupo sobrepeso/obesidade foi $94,4 \pm 4,3$ (IC95\%: 93,4 a 95,5) e para o subgrupo de crianças eutróficas foi 45,3 $\pm 24,2$ (IC95\%: 41,7 a 48,9). Quando avaliados os percentis de IMC segundo o gênero, observou-se: percentil médio de sobrepeso/obesidade masculino 94,4 \pm 4,7 e feminino $94,5 \pm 3,8$, não havendo diferença entre ambos.

\section{Discussão}

Este estudo avaliou a prevalência de sobrepeso/obesidade e eutrofia em crianças e adolescentes com constipação crônica funcional. Conforme dados mostrados na Tabela 1, observouse que os valores da média e do intervalo de confiança da média denotam homogeneidade entre os dois subgrupos, tanto nas características sociodemográficas quanto clínicas, sugerindo que tais variáveis não influenciaram na determinação da prevalência de sobrepeso/obesidade.

A prevalência de constipação intestinal na infância ${ }^{(19)}$ e a de sobrepeso/obesidade ${ }^{(4)}$ têm aumentado nas últimas décadas. Assim, Freed et al ${ }^{(20)}$, comparando os anos de 1993 e 2001, demonstraram aumento no número de consultas de pacientes com constipação intestinal, tanto por pediatras generalistas quanto por especialistas. No presente estudo, encontrou-se prevalência de $24,4 \%$ de sobrepeso/obesidade em crianças com constipação crônica funcional, sendo que a prevalência da obesidade, em três estudos recentes no Brasil ${ }^{(21-23)}$, variou de 20 a $34 \%$. Portanto, a prevalência de sobrepeso/obesidade encontrada em crianças com constipação crônica funcional neste estudo está de acordo com a prevalência esperada para a população pediátrica brasileira.

Os resultados obtidos nesta pesquisa divergem de Pashankar e Loening-Baucke ${ }^{(12)}$ e Misra et a ${ }^{(13)}$. Esses estudos mostraram maior prevalência de sobrepeso/obesidade em crianças com constipação funcional em comparação aos grupos controle. Entretanto, Pashankar e Loening-Baucke ${ }^{(12)}$ utilizaram grupo controle com prevalência de sobrepeso/ obesidade menor do que aquela referida para a população geral pediátrica americana ${ }^{(24,25)}$, o que poderia justificar tais

Tabela 1 - Variáveis sociodemográficas e clínicas das crianças dos subgrupos: eutróficas e sobrepeso/obesidade.

\begin{tabular}{lccc}
\hline & Eutróficas* & Sobrepeso/obesidade* & $\boldsymbol{p}$ \\
\hline Sexo masculino $n$ (\%) & $97(50,5 \%)$ & $34(49,3)$ & $\mathrm{ns}$ \\
Idade crianças (meses) & $75,4 \pm 33,6(70,8-80,2)$ & $80,5 \pm 31(72,7-88,4)$ & $\mathrm{ns}$ \\
Idade mãe (anos) & $30,3 \pm 6,7(29,0-30,9)$ & $31,2 \pm 6,5(29,6-32,9)$ & $\mathrm{ns}$ \\
Idade pai (anos) & $34,7 \pm 9(33,3-36,0)$ & $35,4 \pm 7,1(33,6-37,2)$ & $\mathrm{ns}$ \\
Escolaridade mãe (anos) & $8,5 \pm 3,2(8-8,9)$ & $7,8 \pm 3,3(7,0-8,7)$ & $\mathrm{ns}$ \\
Escolaridade pai (anos) & $8,4 \pm 3,2(7,9-8,9)$ & $7,5 \pm 3,6(6,6-8,4)$ & $\mathrm{ns}$ \\
Número de cômodos no domicílio & $4,7 \pm 1,6(4,4-4,9)$ & $5,6 \pm 2,3(5-6,2)$ & $\mathrm{ns}$ \\
Número de pessoas no domicílio & $4,5 \pm 1,7(4,2-4,7)$ & $4,5 \pm 1,4(4,1-4,8)$ & $\mathrm{ns}$ \\
Número de crianças no domicílio & $2,1 \pm 1,2(1,9-2,3)$ & $2,0 \pm 1(1,7-2,2)$ & $\mathrm{ns}$ \\
Tempo de constipação (meses) & $50,2 \pm 34,9(45,2-55,2)$ & $52,4 \pm 30,5(44,7-60,1)$ & $\mathrm{ns}$ \\
\hline
\end{tabular}

*Valores descritos em média, desvio padrão e (intervalo de confiança 95\%); ns: não significante. 
resultados, ressaltando-se ainda que a prevalência de sobrepeso/obesidade neste estudo e no estudo desses autores foi semelhante: 24,4 e $22,4 \%$, respectivamente.

Tanto a constipação quanto a obesidade resultam em problemas comportamentais e/ou psicossociais, o que pode acarretar, por sua vez, um comprometimento da qualidade de vida ${ }^{(26-28)}$. Tais questões criam uma necessidade inusitada no processo de abordagem terapêutica: é possivel haver uma internalização de sintomas como ansiedade, depressão e problemas de adaptação social, que podem levar à externalização, com transtornos do apetite ${ }^{(29)}$. O sobrepeso/ obesidade em crianças com constipação pode ser resultado

\section{Referências bibliográficas}

1. Clayden GS, Keshtgar AS, Carcani-Rathwell I, Abhyankar A. The management of chronic constipation and related faecal incontinence in childhood. Arch Dis Child Educ Pract 2005;90:58-67.

2. Morais MB, Maffei HV. Constipation. J Pediatr (Rio J) 2000;76 (Suppl 1): S147-56.

3. Machado NC, Carvalho MA. Constipação crônica na infância: quanto estamos consultando em gastroenterologia pediátrica? Rev Paul Pediatr 2007;25:114-8

4. Ogden CL, Carroll MD, Curtin LR, McDowell MA, Tabak CJ, Flegal KM. Prevalence of overweight and obesity in the United States, 1999-2004. JAMA 2006;295:1549-55.

5. Martorell R, Khan LK, Hughes ML, Grummer-Strawn LM. Overweight and obesity in preschool children from developing countries. Int J Obes 2000;24: 959-67.

6. Sokol RJ. The chronic disease of childhood obesity: the sleeping giant has awakened. J Pediatr (Rio J) 2000;136:711-3.

7. Dietz WH. Health consequences of obesity in youth: childhood predictors of adult disease. Pediatrics 1998;101:518-25.

8. Delgado-Aros S, Locke GR 3rd, Camilleri M, Talley NJ, Fett S, Zinsmeister AR et al. Obesity is associated with increased risk of gastrointestinal symptoms: a population-based study. Am J Gastroenterol 2004;99:1801-6.

9. Pecora P, Suraci C, Antonelli M, De Maria S, Marrocco W. Constipation and obesity: a statistical analysis. Boll Soc Ital Biol Sper 1981;57:2384-8.

10. Talley NJ, Howell S, Poulton R. Obesity and chronic gastrointestinal tract symptoms in young adults: a birth cohort study. Am J Gastroenterol 2004;99: 1807-14.

11. Fishman L, Lenders C, Fortunato C, Noonan C, Nurko S. Increased prevalence of constipation and fecal soiling in a population of obese children. J Pediatr (Rio J) 2004;145:253-4.

12. Pashankar DS, Loening-Baucke V. Increased prevalence of obesity in children with functional constipation evaluated in an academic medical center. Pediatrics 2005;116:377-80.

13. Misra S, Lee A, Gensel K. Chronic constipation in overweight children. JPEN J Parenter Enteral Nutr 2006;30:81-4

14. Rasquin-Weber A, Hyman PE, Cucchiara S, Fleisher DR, Hyams JS, Milla PJ et al. Childhood functional gastrointestinal disorders. Gut 1999;45 (Suppl 2):II60-8.

15. Barros FC, Victora CG. Exame físico e antropometria. In: Barros FC, Victora CG. Epidemiologia da saúde infantil. São Paulo: Hucitec-Unicef; 1998. p.89-124. de fatores dietéticos, atividade física e influências hormonais e, portanto, necessita de uma melhor avaliação ${ }^{(12)}$. Assim, a fibra alimentar, como terapêutica primordial no tratamento da constipação, poderia ser benéfica também na abordagem do sobrepeso/obesidade pela redução do apetite, aumento da saciedade, aumento do tempo de mastigação dos alimentos e redução da absorção de lipídeos ${ }^{(30)}$.

Portanto, dada a complexidade dos dois problemas individualmente, torna-se fundamental uma atuação multidisciplinar, em especial quando ambos estão presentes de modo simultâneo no mesmo indivíduo, tal como ocorreu em $25 \%$ dos pacientes deste estudo.

16. Centers for Disease Control and Prevention [homepage on the Internet]. Epi Info 2002. Atlanta: CDC; 2002 [cited 2006 Jan 26]. Available from: http://www.cdc.gov

17. Barlow SE, Dietz WH. Obesity evaluation and treatment: expert committee recommendations. Pediatrics 1998;102:E29.

18. GraphPad Prism [homepage on the internet]. GraphPad Prism Version 3.0, 1999 [cited 2006 Jan 26]. Available from: http://www.graphpad.com/prism/Prism.htm

19. Sonnenberg A, Koch TR. Physician visits in the United States for constipation: 1958 to 1986. Dig Dis Sci 1989;34:606-11.

20. Freed GL, Nahra TA, Wheeler JR. Which physicians are providing health care to America's children? Trends and changes during the past 20 years. Arch Pediatr Adolesc Med 2004;158:22-6.

21. Balaban G, Silva GA. Overweight and obesity prevalence in children and adolescents from a private school in Recife. J Pediatr (Rio J) 2001;77:96-100.

22. Giugliano R, Melo AL. Diagnosis of overweight and obesity in schoolchildren: utilization of the body mass index international standard. J Pediatr (Rio J) 2004;80:129-34.

23. Almeida CA, Pinho AP, Ricco RG, Elias CP. Abdominal circumference as an indicator of clinical and laboratory parameters associated with obesity in children and adolescents: comparison between two reference tables. J Pediatr (Rio J) 2007;83:181-5.

24. Hedley AA, Ogden CL, Johnson CL, Carroll MD, Curtin LR, Flegal KM. Prevalence of overweight and obesity among US children, adolescents and adults, 1999-2002. JAMA 2004;291:2847-50.

25. Ogden CL, Yanovski SZ, Carroll MD, Flegal KM. The epidemiology of obesity. Gastroenterology 2007;132:2087-102.

26. Strauss RS. Childhood obesity and self-esteem. Pediatrics 2000;105:e15.

27. Swallen KC, Reither EN, Haas SA, Meier AM. Overweight, obesity, and healthrelated quality of life among adolescents: the national longitudinal study of adolescent health. Pediatrics 2005;115:340-7.

28. Faleiros FT, Machado NC. Assessment of health-related quality of life in children with functional defecation disorders. J Pediatr (Rio J) 2006;82:421-5.

29. Geist R, Grdisa V, Otley A. Psychosocial issues in the child with chronic conditions. Best Pract Res Clin Gastroenterol 2003;17:141-52.

30. Aggett PJ, Agostoni C, Axelsson I, Edwards CA, Goulet O, Hernell O et al. Nondigestible Carbohydrates in the Diets of Infants and Young Children: A Commentary by the ESPGHAN Committee on Nutrition. J Pediatr Gastroenterol Nutr 2003;36:329-37. 